\title{
Scaling properties of the hot gas in early-type galaxies
}

\author{
Trevor J. Ponman ${ }^{1}$ and Ewan J. O'Sullivan ${ }^{1,2}$ \\ ${ }^{1}$ School of Physics \& Astronomy, University of Birmingham, Birmingham B15 2TT, UK. \\ ${ }^{2}$ Harvard-Smithsonian Center for Astrophysics, 60 Garden St, Cambridge, MA02138, USA \\ email: tjp@star.sr.bham.ac.uk
}

Abstract. We examine the processes responsible for the large scatter in the X-ray/optical luminosity scaling relation of early-type galaxies.

\section{The $L_{X}-L_{\mathrm{opt}}$ relation in early-type galaxies}

Optically brighter galaxies tend to have higher X-ray luminosity. The largest sample yet presented, by Ellis \& O'Sullivan (2006), is shown in Fig. 1. It is apparent that: (a) there is a factor $\sim 100$ scatter in $L_{X}$ around the mean relation; (b) the relation steepens at high $L_{B}$, where most galaxies lie well above the discrete source line - their X-ray emission dominated by a diffuse hot gas component; (c) many of these luminous galaxies are the bright central members of groups or clusters (BGGs or BCGs). Here we briefly examine a number of possible causes for the large scatter apparent in this relation.

\section{Sources of scatter in $L_{X}-L_{\mathrm{opt}}$}

Star formation history - Recent star formation can considerably raise the blue luminosity of a galaxy, at a given stellar mass. However, Ellis \& O'Sullivan (2006) showed that replacing $L_{B}$ by K-band luminosity in Fig.1, results in a very similar scatter.

X-ray data quality - The X-ray data used by Ellis \& O'Sullivan (2006) came from ROSAT. However, smaller samples of higher quality datasets from Chandra and XMM (e.g. Diehl \& Statler (2007), Sun (2009)) show similar scatter in the relation.

Galaxy age - It has been recognised for some years (e.g. O'Sullivan et al. (2001)) that spectroscopically young ellipticals have low X-ray luminosity. The compilation of Sansom et al. (2006) shows a decline in $L_{X} / L_{B}$ for 1-2 Gyr after a substantial burst of star formation (e.g. following a galaxy merger), presumably due to hot gas being dispersed by the energetic galaxy winds known to be associated with starbursts. However, galaxies with larger spectroscopic ages show no age trend, and a wide scatter in $L_{X} / L_{B}$.

Galaxy environment - Ellis \& O'Sullivan (2006) found no clear environmental trend in $L_{X} / L_{B}$, apart from the high values apparent in BGGs and BCGs. However, recent studies with Chandra, which is better able to resolve galaxy emission, show that $L_{X} / L_{B}$ is suppressed by a factor 3-4 in non-central galaxies within both clusters (Sun et al. (2007)) and groups (Jeltema et al. (2008)). This presumably results from stripping of hot gas within dense environments. The properties of isolated early-type galaxies provide a probe of whether environmental effects can account for the bulk of the scatter in $L_{X} / L_{B}$. Memola et al. (2009) assembled $L_{X}-L_{B}$ data for 27 isolated ellipticals, and found a good deal of scatter. However, excluding probable 'fossil groups' (Ponman et al. (1994)) and upper limits, the remaining sample is small. Preliminary results from a Chandra study of isolated ellipticals currently underway indicate (Humphrey private communication) that significant $L_{X} / L_{B}$ scatter is still present, but may be limited to a factor $\sim 10$.

Halo mass - Mathews et al. (2006) suggested that the primary factor driving $L_{X}$ is the mass of the dark halo surrounding a galaxy. For a sample of ellipticals (mostly BGGs) they found that $L_{X} / L_{K}$ increased with X-ray derived virial mass. This is consistent with the earlier result, that 


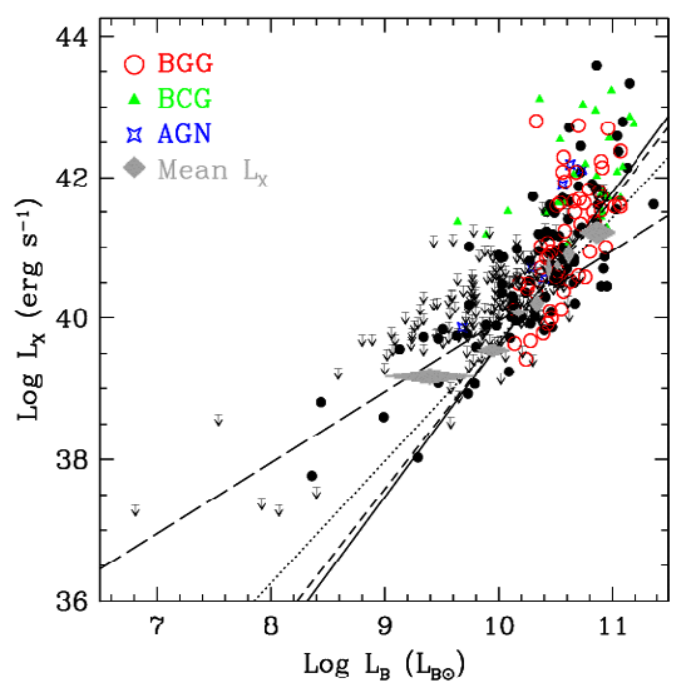

Figure 1. $L_{X}-L_{B}$ plot for 401 early-type galaxies from Ellis \& O'Sullivan (2006). The long dashed line is the discrete source contribution estimated by Kim \& Fabbiano (2004), other lines show fits to various subsets of the sample, and the grey diamonds represent the mean $L_{X}$ in a series of $L_{B}$ bins.

BGGs have $L_{X} / L_{B}$ ratios much higher than other elliptical galaxies. The study of NGC 4555 by O'Sullivan \& Ponman (2004) shows that isolated ellipticals can also possess substantial dark matter halos.

Feedback - For a given dark halo mass, the gas content can be affected by feedback processes. Injection of energy raises the entropy, resulting in low density and X-ray luminosity. The low $L_{X} / L_{B}$ ratio seen in ellipticals with recent star formation is probably an example of this. AGN feedback may have similar effects, if it can couple effectively to the surrounding gas. Sun (2009) finds that all radio-bright elliptical galaxies contain hot gas coronae - either compact or extensive. He postulates that AGN jets can couple to the extended hot halos found in groups or clusters, but not to compact gas cores.

\section{References}

Diehl S. \& Statler T. S. 2007, ApJ 668, 150

Ellis S. J. \& O'Sullivan E. J. 2006, MNRAS 367, 627

Jeltema T. E. et al. 2008, ApJ 679, 1162

Kim D.-W. \& Fabbiano G. 2004, ApJ 611, 846

Mathews W. G. et al. 2006, ApJ 652, L17

Memola E. et al. 2009, A\& $A$ 497, 359

O'Sullivan E. J., Forbes D. A., \& Ponman T. J. 2001, MNRAS 324, 420

O’Sullivan E. J. \& Ponman T. J. 2004, MNRAS 354, 935

Ponman T. J. et al. 1994, Nature 369, 462

Sansom A. et al. 2006, MNRAS 370, 1541

Sun M. et al. 2007, ApJ 657, 197

Sun M. 2009, arXiv:0904.2006 